\title{
Dynamics of the solar photosphere with IBIS
}

\section{Reversed intensity structure in the mid-photosphere}

\author{
K. Janssen and G. Cauzzi
}

INAF - Osservatorio Astrofisico di Arcetri, Largo Enrico Fermi 5, 50125 Firenze, Italy

e-mail: kjanssen@arcetri.astro.it

Received 5 October 2005 / Accepted 16 December 2005

\section{ABSTRACT}

Context. We investigate the quiet solar photosphere with the new Interferometric BI-dimensional Spectrometer (IBIS), recently installed at the Dunn Solar Telescope of the National Solar Observatory, Sacramento Peak, NM.

Aims. We study the occurrence of reverse granulation in the mid-photosphere, and its properties.

Methods. IBIS, a double Fabry-Perot system with a narrow passband of $20 \mathrm{~m} \AA$ at $6000 \AA$, was used to acquire a one hour sequence of $2 \mathrm{D}$ scans in the photospheric Fe I 7090.4 $\AA$ and Fe II $7224.5 \AA$ lines, and in the chromospheric Ca II $8542 \AA$ line. Photospheric velocities are measured for statistical study. To visualize the reversed intensity structure, the influence of velocity on the steep photospheric profiles is taken into account. The correlation between mid-photospheric intensities and continuum is then studied, considering the effects of spatial smoothing and time delay. The temporal evolution of the cross-correlation coefficients is analyzed.

Results. The distribution of photospheric velocities is consistent with values in the literature, thus confirming the reliability of the instrument for this kind of study. "Reversed granulation" is clearly visible in the Fe I $7090.4 \AA$ line center, formed at about $200 \mathrm{~km}$. It provides a weak anti-correlation with the continuum, as expected from numerical simulations. Images acquired in the far wings of the Ca II line display the same pattern with a slightly higher correlation. In both cases a spatial smoothing larger than $0.5^{\prime \prime}$ decreases the level of anti-correlation, while a time delay of about 2 min increases it. Together, these facts suggest that the phenomenon is mainly due to a reversal of temperature fluctuations between granules and intergranules, occurring in the mid-photosphere. The temporal evolution of the anti-correlation between the Fe I line center and the continuum reveals a clear oscillatory signal that we consider as highly suggestive of the presence of gravity waves developing from the mid-photosphere outwards.

Key words. Sun: photosphere - Sun: granulation - line: profiles

\section{Introduction}

Understanding the structure and dynamics of the quiet solar photosphere is fundamental for a full comprehension of granulation in late-type stars, and of convection in general. In turn, these are important for other solar and general astrophysical problems, from the structure and possible thermal bifurcation of the chromosphere (Wedemeyer et al. 2004) to coronal heating due to shuffling of magnetic fields by granular motion (Peter et al. 2004) to the precise determination of elemental abundances (Shchukina \& Trujillo Bueno 2001; Asplund et al. 2000b). The recent advent of suitable adaptive optics devices (Rimmele 2004), of efficient post-facto reconstruction techniques (Janssen 2003; Löfdahl 2002), and of high throughput instruments, makes it now feasible to obtain the necessary consistent, long-duration, and spectrally resolved high resolution observations.

As a first paper in a series dealing with the dynamics of the lower solar photosphere, we present here initial results obtained with a new 2D spectrographic device, the Interferometric
BI-dimensional Spectrometer (IBIS, Cavallini 2006), that can satisfy such demanding instrumental constraints. After a brief description of the instrumental characteristics and the data acquisition (Sect.2), we review some "classical" properties of the non-magnetic photosphere, comparing intensity and velocity distributions with the pertinent literature (Sect. 3). We then study reversed granulation (Sect. 4) in the middle photosphere.

Several observational studies utilizing inversion of spectroscopic data report the reversal of the temperature difference between granules and intergranular lanes in the low- to mid-photosphere (Rodríguez Hidalgo et al. 1999; Borrero \& Bellot Rubio 2002; Berrilli et al. 2002; Puschmann et al. 2003). Such a reversal is present in numerical simulations of the surface convection as well (Nordlund 1985 and following work; Wedemeyer et al. 2004), mainly due to the adiabatic expansion cooling (or compression heating) of the overshooting plasma. A clear, visual demonstration of the phenomenon is provided by the wispy intensity pattern that has been observed in the extended wings of the Ca II H \& $\mathrm{K}$ lines (e.g. Fig. 3 in Lites et al. 1999; Rutten et al. 2004), and is often called "reversed 
granulation". This intensity pattern displays a partial anticorrelation with the underlying continuum granulation pattern, as first diagnosed by Evans \& Catalano (1972), and explained by Nordlund (1985) as due to the cooling of gas penetrating into a stable layer. However, as the latter author points out, because of the temperature fluctuations associated with the horizontal motion in the granular pattern, such a negative correlation must be weak. Rutten et al. (2004) analyze broadband Ca II H wing images as a signature of the mid photosphere and find an average anti-correlation with the G-band intensity (as a proxy for the blue continuum) of about 0.6 , after optimizing for spatial resolution and temporal delay. They estimate that a fraction of the mid-photospheric intensity pattern is ascribable to the presence of gravity waves that should be easily excited by the same convective overshoot that generates the temperature reversal. Higher up in the atmosphere, at about $400 \mathrm{~km}$ where TRACE UV continua form, the temperature reversal plays a minor role and the intensity pattern is mostly set by gravity wave interference (Rutten \& Krijger 2003).

Despite the fact that reversed granulation is obviously photospheric in origin, the many observational studies conducted on granulation utilizing narrowband imaging instruments in photospheric lines (Salucci et al. 1994; Espagnet et al. 1995; Hirzberger 2002) never show the wispy intensity pattern described above. One reason is that, while the broad wings of the $\mathrm{Ca}$ II $\mathrm{H} \& \mathrm{~K}$ lines have a very reduced sensitivity to velocity, and thus the observed intensity pattern is mostly set by the temperature structure, this is not true for photospheric lines. Indeed, given the typical amplitude of the convective motions, any monochromatic image acquired within a photospheric line will actually display mixed behavior, where radiation produced at the nominal wavelengths can lie beside radiation emitted in the continuum or core levels, i.e. at heights displaying a rather different temperature structure. This effect is more pronounced the higher the spatial resolution achieved, as exemplified by the variety of granular profiles in the simulations of Asplund et al. (2000a). A further reason is the spectral passband of imaging instruments that often amounts to a large fraction of the photospheric lines' widths (e.g., Hirzberger 2002): this can mix the information from layers structured by very different temperature patterns in a single image. In the following, we take advantage of the high spectral purity, as well as of the multiple line diagnostics offered by IBIS, to investigate these issues.

\section{Observation \& data reduction}

\subsection{Instrumental characteristics}

A detailed description of the IBIS instrument can be found in Cavallini (2002, 2006), here we briefly recall its basic characteristics. The Interferometric BI-dimensional Spectrometer (IBIS) is installed at the Dunn Solar Telescope of the National Solar Observatory (NSO/DST), USA, and fed through the High Order Adaptive Optics system (Rimmele 2004). The central elements of IBIS are two air-spaced Fabry Perot Interferometers (FPI), $50 \mathrm{~mm}$ in diameter, mounted in classical configuration, that provide an Airy function of $20-40 \mathrm{~m} \AA F W H M$ in the spectral range of $5800 \AA-8600 \AA$. Currently, there are five prefilters
Table 1. Observational details for the spectral lines used. $g$ is the Landé factor.

\begin{tabular}{ccccc}
\hline \hline Spectral Lines & $\lambda$ & \# Positions & Mean Stepsize & $g$ \\
\hline Fe II & $7224.5 \AA$ & 17 & $25 \mathrm{~m} \AA$ & 0 \\
Fe I & $7090.4 \AA$ & 16 & $30 \mathrm{~m} \AA$ & 0 \\
Ca II & $8542 \AA$ & 27 & $116 \mathrm{~m} \AA$ & 1.1 \\
\hline
\end{tabular}

with $F W H M$ of $3 \AA$ to $5 \AA$ available as order sorters, centered around the following spectral lines: $\mathrm{NaD}_{1} 5896 \AA$; the magnetic Fe I lines at 6301-6302 $\AA$; the magnetically insensitive Fe I 7090.4 ̊ and Fe II 7224.5 ̊ lines; and Ca II $8542 \AA$.

Various relay optics display a $80^{\prime \prime}$ diameter, circular section of the Sun on a $1024 \times 1024$ Princeton CCD, with a spatial scale of $\sim 0.08^{\prime \prime} /$ pixel. As the two FPIs are mounted in a collimated beam, images are not strictly monochromatic, but suffer a shift to shorter wavelengths towards the border of the FOV. This instrumental blueshift scales quadratically with radial distance from the center of the FOV and ranges from $60 \mathrm{~m} \AA$ at $6000 \AA$ to about $100 \mathrm{~m} \AA$ at $8500 \AA$ at the outermost pixels.

Typical exposure times vary between 10 and $40 \mathrm{~ms}$. The interferometers' tuning from one wavelength to the next takes less than $20 \mathrm{~ms}$, while switching the prefilters by rotating the filter wheel takes 1-2 s. The final acquisition rate is dominated by the read-out time of the CCD camera, and amounts to 2.5 images $^{-1}$ at full image size, and 4 images $\mathrm{s}^{-1}$ at an on-chip binning of $2 \times 2$. A two dimensional scan through a spectral line using 15-20 spectral positions thus requires about 4-8 s. IBIS is equipped with a white light channel that provides broad-band images with a $50 \AA$ passband around $7000 \AA$ strictly simultaneous to the narrow-band ones.

\subsection{Data acquisition and reduction}

The data studied in this paper was acquired on June 2nd, 2004 on a quiet area at disk center. The observing campaign aimed at covering a large atmospheric range, therefore each data sequence contains scans of two photospheric as well as a chromospheric line, see Table 1 . The images were binned $2 \times 2$, providing a three-dimensional data cube with two spatial dimensions of $512 \times 512$ pixels $\left(0.16^{\prime \prime}\right.$ pixelsize $)$, and one spectral dimension.

The exposure time of a single frame was $25 \mathrm{~ms}$, providing a $\mathrm{S} / \mathrm{N}$ ratio of about 150 in the continuum, and the whole cycle was repeated every $19 \mathrm{~s}$. A total of 171 sequences were acquired during good seeing conditions (power spectra analysis gave a spatial resolution of $\approx 0.4^{\prime \prime}$ for the continuum images), covering a time span of $54 \mathrm{~min}$. About 100 flat field sequences were acquired in analogy to the data, but using a defocused, rapidly moving telescope setting.

Dark and gain corrections were applied to the data. We note that, since the original images are not strictly monochromatic, special care must be taken in deriving a proper gain table, free of unwanted spectral information, i.e. from intensity variations due to the actual spectral line, rather than to the optical setup and detector response. Details about the procedure will be provided in a forthcoming paper. All scans are tracked in time by 

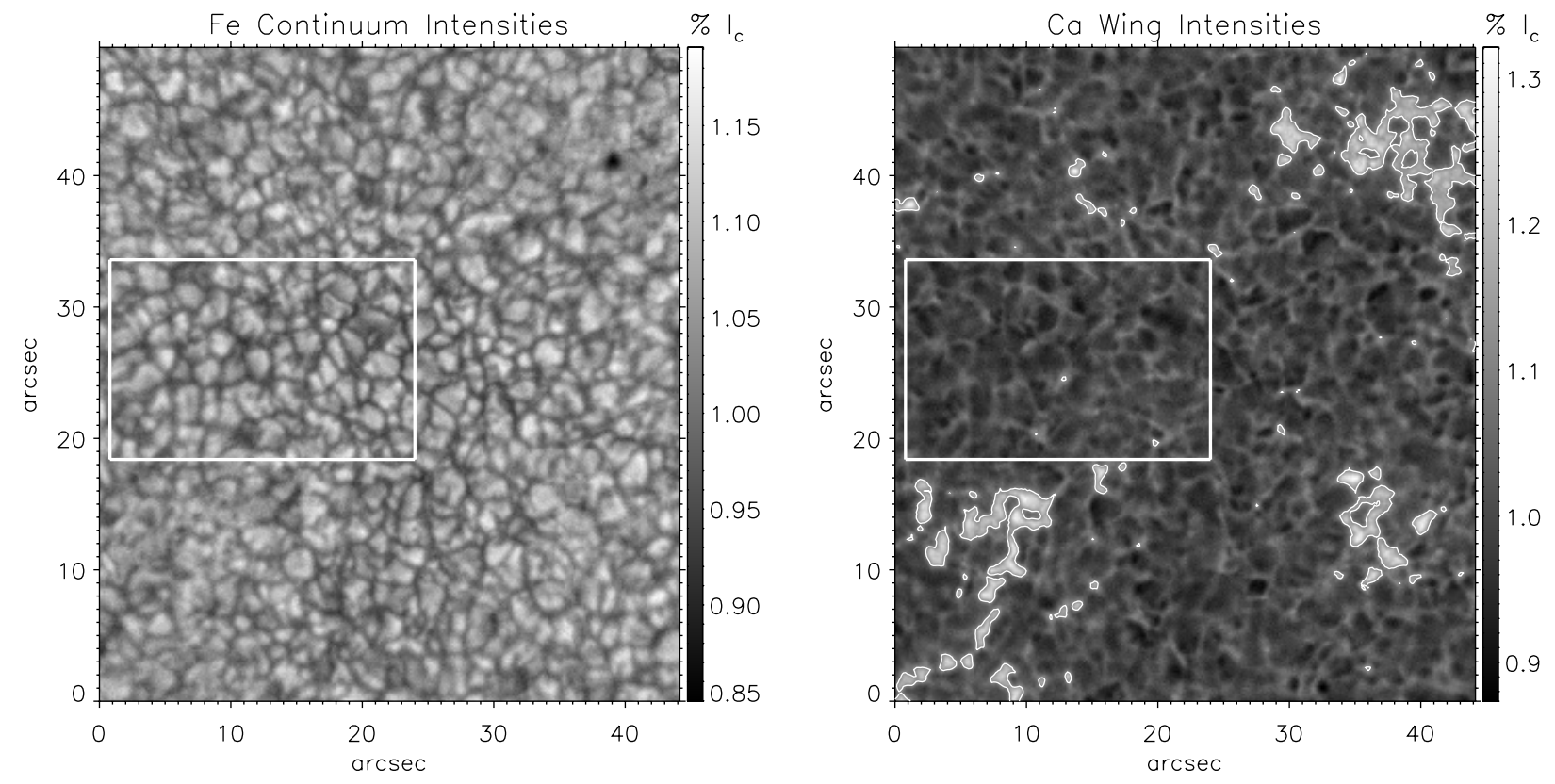

Fig. 1. Common FOV for all scans and spectral lines within the 54 min sequence (cut from the original IBIS data of $80^{\prime \prime}$ diameter). Left: continuum near the Fe I 7090.4 A line. Distorted granulation in the image corners indicates the presence of magnetic (network) areas. Right: blue wing of Ca II $8542 \AA, 0.9 \AA$ from the line core. At this wavelength bright structures clearly outline magnetic activity. The white rectangle indicates a very quiet region, which is used in the remainder of the paper.

correlating the continuum images of subsequent scans, and the common FOV is extracted, as shown in Fig. 1. Although not strictly necessary for the computation of line parameters such as velocity, the original data is interpolated using the blueshift map in order to produce monochromatic images, as this facilitates the comparison of intensity patterns at different heights in the atmosphere (see Sect. 4). A subsonic filter is applied to the time series of each monochromatic image (i.e. spectral position) separately, to remove the influence of 5-min oscillations. We follow a commonly used scheme (e.g. Rutten et al. 2004) and cut a cone out of the $k_{x}-k_{y}-\omega$ space whose outer borders correspond to a sound velocity of $6 \mathrm{~km} \mathrm{~s}^{-1}$ in the photosphere.

\subsection{Choice of lines and FOV}

In the following, we will mainly analyze the Fe I 7090.4 A data. This line has an excitation potential of $4.23 \mathrm{eV}$, and should therefore have a low temperature sensitivity (Nesis et al. 1999), whereas the steep wings give it a strong sensitivity to velocity (Cabrera Solana et al. 2005). In semi-empirical models of the quiet Sun such as VAL-C, its line core forms around $200 \mathrm{~km}$ above the continuum-forming layers. However, synthesis of the line in snapshots of 3D hydrodynamical simulations such as those of Stein \& Nordlund (1998) show that the line can sample higher layers, at least up to $300 \mathrm{~km}$ (Carlsson 2004, private communication; Asensio Ramos et al. 2006). So Fe I $7090.4 \AA$ should be able to probe a large fraction of the photosphere, at least up to where the inversion of contrast between granular and intergranular features is expected (see Sect. 4). Finally, in the absence of polarimetric measures, the use of a magnetically insensitive line guarantees that any modification of the spectral profile is to be ascribed to thermodynamical and kinematic properties only, rather than to small, strong magnetic concentrations such as those described by Domínguez Cerdeña et al. (2003).

Both Fe II $7224.5 \AA$ and Ca II $8542 \AA$ data will be utilized to confirm, or constrain, properties derived from the Fe I 7090.4 line. The Fe II line, formed in the deep photosphere, will be used mainly together with the Fe I line to infer possible variations with height in the line-of-sight velocities. The contribution function for the broad wings of the Ca II line peaks in the mid-photosphere, while for a narrow range of wavelengths around the core the formation reaches up to the midchromosphere (Uitenbroek 1989; 2004, private communication).

The CaII line is sensitive to the presence of magnetic structures, so the intensity measured in the extended wings, from about \pm 0.7 to $\pm 1.2 \AA$ from line core, is a faithful tracer of the network features (see Fig. 1 and Cauzzi et al. 2006), much as the case of the Ca II H \& $\mathrm{K}$ lines. We use this characteristic to define a very quiet region within the field of view, avoiding the areas brighter than two sigma above average intensity at $0.9 \AA$ in the blue wing. The white box in Fig. 1 outlines this pure "internetwork" region that will be used throughout the paper, as in the case analyzed by Rutten et al. (2004).

\subsection{Extraction of velocities}

Line of sight velocities are calculated in terms of center of gravity $(\mathrm{COG})$ and line core velocities from the spectral profiles of Fe I 7090.4 $\AA$ and Fe II $7224.5 \AA$, hereafter referred to as $v_{\mathrm{COG}}^{7090}$, $v_{\text {core }}^{7090}$, etc. The line core velocity, calculated as the wavelength of the minimum of the parabola that goes through the three points around the lowest intensity of a line profile, probes the 
Table 2. Average values and standard deviation of line-of-sight velocities determined for the two photospheric lines, with two different methods. All values are in $\mathrm{m} \mathrm{s}^{-1}$, averaged over the quiet area in all time steps (2.4 million pixels). Negative values indicate upflow.

\begin{tabular}{ccccc}
\hline \hline Velocities & $v_{\text {COG }}^{7224}$ & $v_{\text {core }}^{7224}$ & $v_{\text {COG }}^{7090}$ & $v_{\text {core }}^{7090}$ \\
& & & & \\
\hline mean & -20 & -70 & -40 & -10 \\
rms & 390 & 480 & 330 & 280 \\
\hline
\end{tabular}

highest atmospheric layers sampled by a spectral line, but tends to fail for weak noisy lines such as Fe II 7224.5 ̊. The COG velocity, instead, determined as the average wavelength of the spectral positions weighted by the line profile, gives more robust results combining all spectral information in one velocity value (Cauzzi et al. 1993), and is insensitive to spectral resolution as long as the sampling of the line is complete and the spectral transmission function is symmetric (Uitenbroek 2003). COG velocities represent an average over the contribution function of the whole line profile, but weighted towards the formation height of the line center by the choice of the intensity profile as the distribution function. Hence for the low photospheric lines chosen, the COG velocities should be very similar to line core velocities (Uitenbroek 2003).

The zero point for all velocities is defined as the average COG velocity over the full FOV and timeseries (15 million values) of each line, as commonly used (see e.g. Berrilli et al. 2002; Hirzberger 2002).

\section{Amplitude and persistence of velocities}

Table 2 gives the line-of-sight velocities and their spread for both photospheric lines and both methods averaged over the whole time series in the quiet internetwork region (2.4 million values). Even though the Fe II $7224 \AA$ line gives noisier results, in particular for the line core velocities, the average velocities for the two lines are consistent. The negative average value of about $-40 \mathrm{~m} \mathrm{~s}^{-1}$ depends on our definition of the zero velocity, since the full FOV includes some magnetic areas that display more positive velocities.

The spread of velocities is more easily comparable with the literature than the average values. The rms yields about $400 \mathrm{~m} \mathrm{~s}^{-1}$ for the deeper Fe II line, and about $300 \mathrm{~m} \mathrm{~s}^{-1}$ for the mid-photospheric Fe I line. These quantities are consistent with the findings of Berrilli et al. (2002) that, employing a similar set of spectral lines, observe a "braking" of convective velocities with height in the first layers of the photosphere. However, our values remain well below the value of $\sim 600 \mathrm{~m} \mathrm{~s}^{-1}$ found by Krieg et al. (2000) at the height of approx. $100 \mathrm{~km}$. The disagreement may have many different causes, such as different image restoring processes, $p$-mode filtering, or sensitivity of the spectral lines used. The $v_{\mathrm{COG}}^{7090}$ velocities listed in Table 2 are displayed in histogram form in Fig. 2 (left) and show a rather asymmetric velocity distribution. The slope for positive velocities (downflows) is much steeper than the slope of negative values. Such a property has been reported by
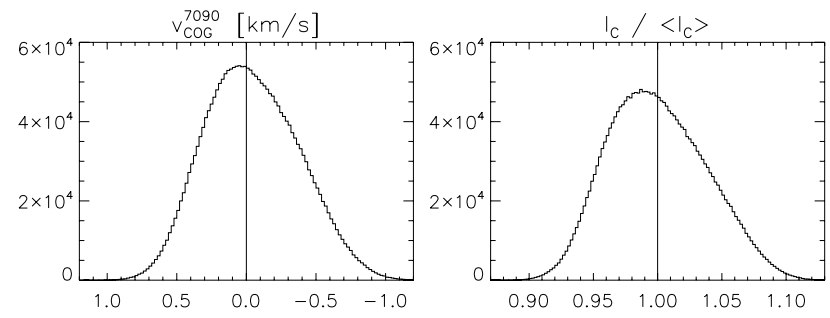

Fig. 2. Histograms of the $v_{\mathrm{COG}}^{7090}$ line of sight velocities (left) and continuum intensities (right) for the quiet area time series (2.4 million values). Positive values indicate downward motion.
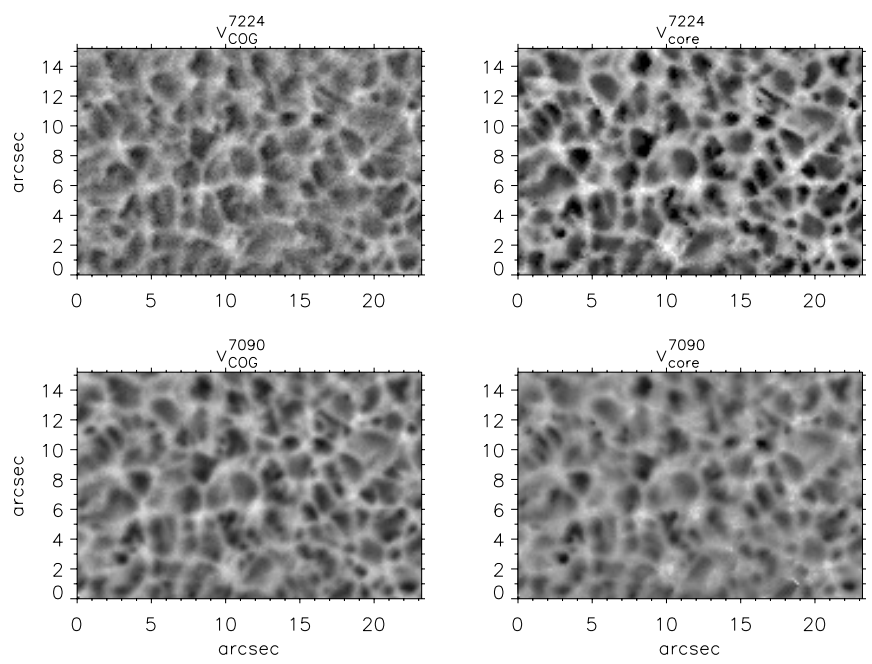

Fig. 3. Maps of line-of-sight velocity calculated for a representative scan in the selected non-magnetic region. Tickmarks are in arcsec. The amplitude scale, $\pm 1.3 \mathrm{~km} \mathrm{~s}^{-1}$, is the same for all maps.

Keil \& Canfield (1978) and Hirzberger (2002), but is much more pronounced in our case.

The normalized continuum intensities (Fig. 2 right) exhibit the same kind of asymmetric distribution as the velocities. Indeed, the correlation between the granular intensity pattern and vertical velocities is very strong in our observations, giving correlation coefficients of around -0.80 for all four velocity maps. An asymmetry comparable to that of Fig. 2 is seen from spectral synthesis in snapshots of 3D numerical simulations, adapted to the observations by appropriate spectral and spatial smearing (Asensio Ramos et al. 2006).

Figure 3 displays the velocity maps obtained, using the two photospheric lines and methods, for a representative scan. The granular pattern is clearly visible (dark corresponds to negative velocities, i.e. upflows) and fairly similar in all the maps, consistent with the known property that velocity structures persist over a large range of heights in the photosphere, contrary to the intensity structures (compare next section). All four velocity maps have strong correlations with one another (Table 3 ), being even stronger the shorter the atmospheric height difference is, as reported by Hanslmeier et al. (2000). Especially high values of 0.9 (Fe II) and 0.95 (Fe I) are found between velocities computed with the two methods for the same line. For the better determined Fe I $7090 \AA$ velocity, we found that the values of the core and COG velocities are comparable (following $v_{\text {core }}=0.8 \cdot v_{\text {COG }}$, see Fig. 4 , left). This confirms that, at least 
Table 3. Correlation coefficients between velocity maps sampling different atmospheric layers (from lower to higher: $v_{\mathrm{COG}}^{7224}, v_{\mathrm{core}}^{7224}, v_{\mathrm{COG}}^{7090}$, $\left.v_{\text {core }}^{7090}\right)$, averaged over the time series (171 scans).

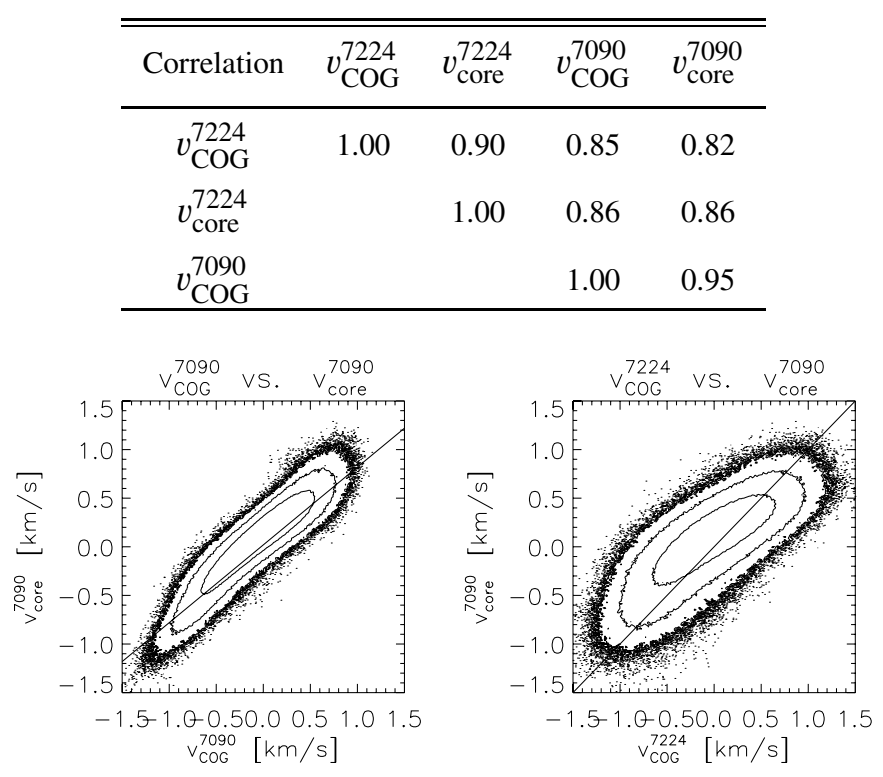

Fig. 4. Scatter plots of quiet region line-of-sight velocities. The curves visualize the density within the plot, as a histogram seen from above. Left: plotted are $v_{\text {core }}^{7090}$ against $v_{\mathrm{COG}}^{7090}$ velocities (2.4 million points) with the regression line of $0.8 \cdot x+0.02$, showing the good agreement of measurements with the two methods within this mid-photospheric line. Right: $v_{\text {core }}^{7090}$ against $v_{\text {COG }}^{7224}$ velocities (2.4 million points) with the bisecting line. The variation of velocity in height is stronger for intergranular flows (positive values) than for granular.

for this low or mid-photospheric line, the line core velocity is equivalent to the velocities derived from the center of gravity, as predicted by Uitenbroek (2003).

Finally, numerical simulations of surface convection, as well as two-component atmospheric models such as that of Borrero \& Bellot Rubio (2002), find that the variation of velocity in height is much larger for intergranules than for granules, where the latter have essentially the same velocity throughout the mid photosphere. A first attempt to verify this in our data is displayed in Fig. 4 (right panel), where we plot the $v_{\text {core }}^{7090}$ velocities as proxies for the highest layers sampled against $v_{\mathrm{COG}}^{7224}$ as an indicator for the lowest photospheric layers. While the granular velocities (negative values) remain closer to the bisecting line, positive values representing the intergranular velocities tend to decelerate with height. A better segregation of structures and detailed analysis of their parameters will be the subject of a forthcoming paper.

\section{Granulation persistence: the "reversed granulation" pattern}

\subsection{Monochromatic images}

In principle, monochromatic images acquired with a narrowband spectrometer scanning through a photospheric line could provide an intensity mapping of the convective overshoot with height, as different parts of a line sample different atmospheric layers. In particular, one should be able to reveal the "reversed granulation" pattern at layers where the inversion of temperature difference between granules and intergranular lanes becomes appreciable, supposedly occurring at about $200 \mathrm{~km}$ above the continuum forming layers. In practice, however, this effect is heavily masked by concurrent influences such as the intrinsic line sensitivity to temperature and velocity fields, the actual width and shape of the contribution function for the spectral line, and the precise shape of the instrument' spatial and spectral passbands (e.g., Al et al. 2002).

This is exemplified by the sequence of spectrograms displayed in Fig. 5 that sample the Fe I $7090 \AA$ line at -60,-30, 0, $+60,+30 \mathrm{~m} \AA$ from nominal line center and at a close continuum. The blue wing images (panels $a$ and $b$ ) show a reversal of the continuum pattern that gets stronger closer to the line center, while the red wing images ( $d$ and $e$ ) display enhanced granulation. The line core itself $(c)$ does not bear any obvious resemblance to the continuum intensities $(f)$. Further in the line wings (not shown in the figure) the monochromatic images produce a smooth transition to normal granulation. In a more quantitative way, these properties are presented in Fig. 7. Here, the correlation between the intensities at each sampled wavelength and the continuum (dotted line) is plotted together with the observed average line profile (dashed line). Anticorrelation with the continuum image is found only in the blue wing of the line, while the correlation for the core position is close to zero.

The chief ingredient of the intensity pattern seen in Fig. 5 and the dotted curve of Fig. 7 is the cross-talk between the velocity and the intensity field, which is especially important for the FeI 7090.4 $\AA$ line. In fact, at any fixed wavelength in the blue wing, the red-shifted intergranular lanes comprise radiation coming from closer to the continuum, thus brighter than the blue-shifted granular intensities that originate closer to the core of the line. As a consequence, we see a pattern of apparent reversed granulation, i.e. whose origin is not a reversal of temperature fluctuations in the granulation structures. The opposite holds in the red line wing: since the intensity in the granules due to their blueshift is even larger than in the redshifted intergranules, enhanced granulation is observed.

A particularly clear example of this effect is given by the feature at the $\left[16^{\prime \prime}, 10^{\prime \prime}\right]$ position in Fig. 5 (indicated by the arrow) that is very bright in the line core image (panel $c$ ). Comparing the wing images, we identify it as a very dark feature in the $-60 \mathrm{~m} \AA$ image, while not obviously discernible in the $-30 \mathrm{~m} \AA$ one. From inspection of the red wing and continuum images, the structure represents a very blueshifted granule with a velocity of $1.2 \mathrm{~km} \mathrm{~s}^{-1}$, clearly visible in the maps of Fig. 3.

This kind of cross-talk effect has been discussed, with particular attention to the red-wing enhanced granulation pattern, by Sütterlin et al. (2001), who used the resonance line of Ba II at $4554 \AA$ for Doppler diagnostics. In their case, as well as here, it is of fundamental relevance that $i$ ) the observations are obtained with high spatial resolution to well resolve the granuleintergranule pattern; $i$ ) the line has a strong sensitivity to velocity; and iii) the instrumental spectral profile is narrow enough not to "dilute" the encoded velocity information. 

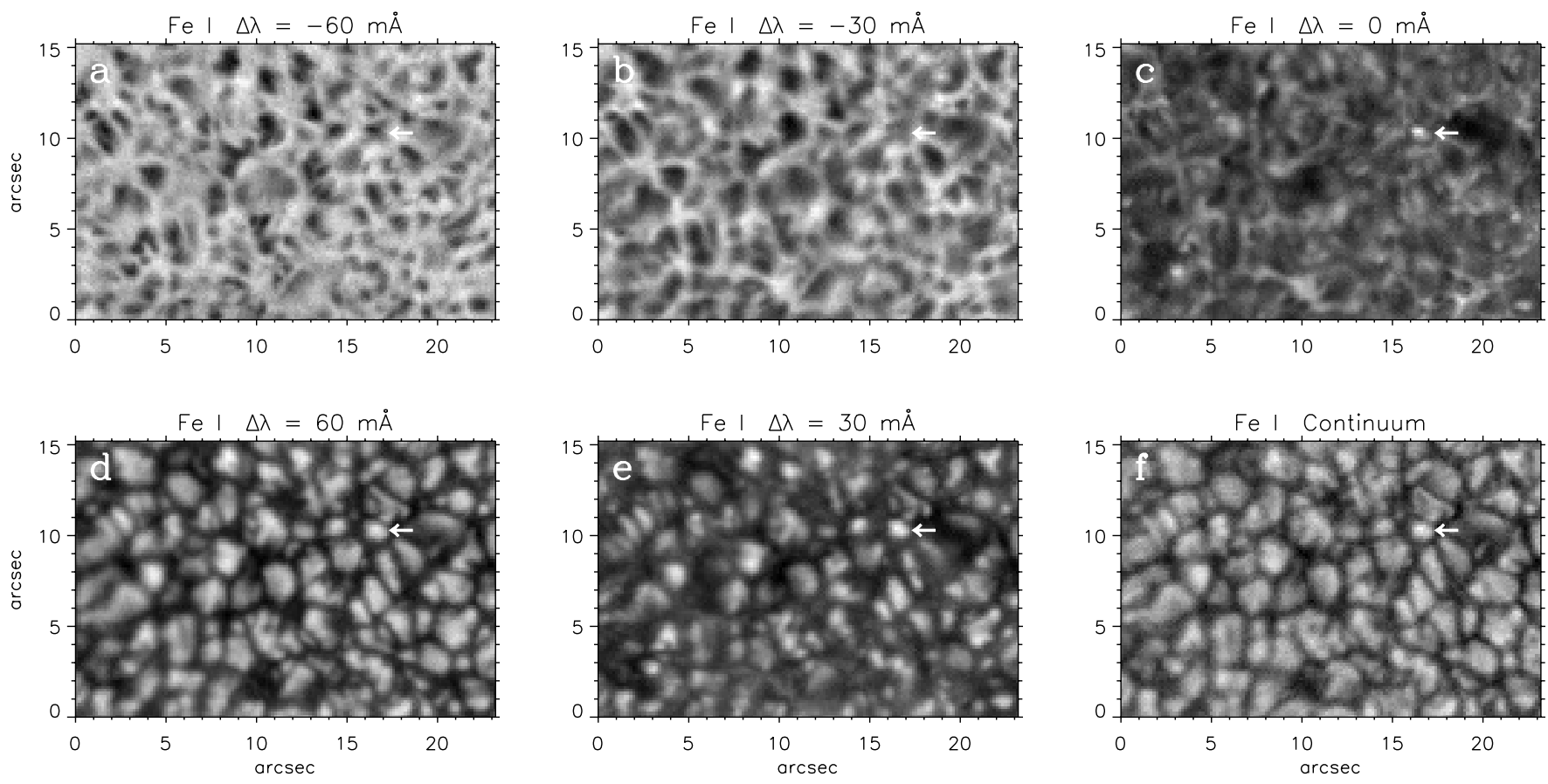

Fig. 5. Intensity maps at different wavelength positions within the $7090.4 \AA$ line, for a representative scan in the quiet area. From left to right, top to bottom: $-60,-30,0+60,+30 \mathrm{~m} \AA$ from nominal line core and continuum intensities. The cross-talk between velocity and intensity is apparent, as granulation appears "reversed" in the blue wing, and "enhanced" in the red wing. The arrows indicate a feature discussed in the text.
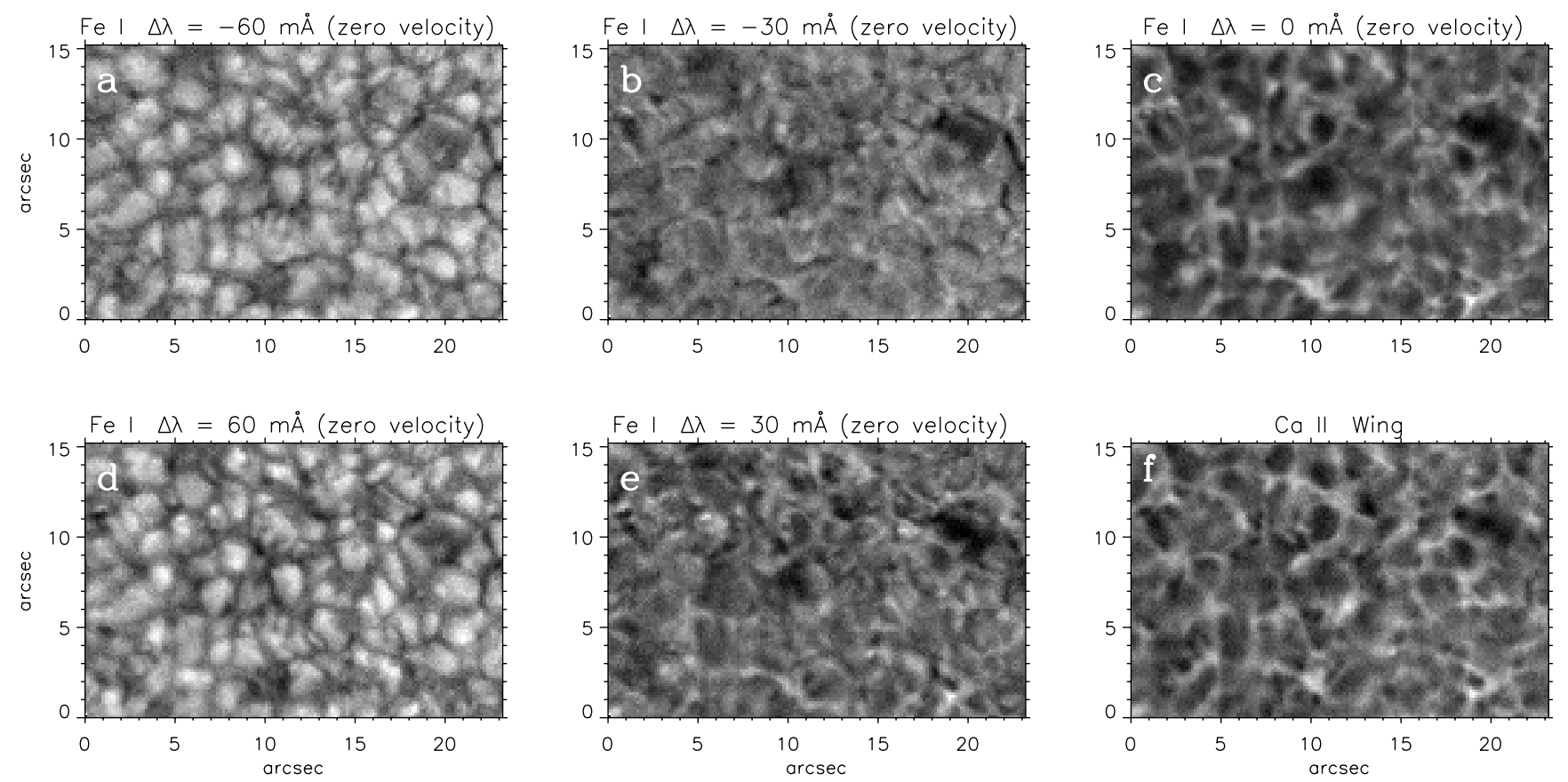

Fig. 6. Panels a)-e): as Fig. 5, after "removal" of velocity, i.e. considering intensity images at given distance from actual line core position of each pixel. Symmetric positions in the wings give essentiallly the same pattern. Panel $f$ here displays co-temporal intensity image at about $0.9 \AA$ in the blue wing of Ca II 8542. The similarity between the actual line core of Fe I 7090 c) and the blue wing of Ca II 8542 f) is remarkable.

\subsection{Uncovering "real" reversed granulation}

The central part of the Fe I $7090 \AA$ A line should form high enough in the photosphere to actually sample the layers where the temperature difference between granules and intergranules reverses (Sect. 2.3). Searching for this signature, we attempt to disentangle the effect of thermal structure from that of the velocity by shifting all spectral profiles according to their (COG) velocity as a first-order correction. Since the vertical velocities do not change much throughout the line formation range (Sect. 3), this corresponds to resampling all of the profiles with the same, at rest, wavelength scale. Barring large line asymmetries introduced by peculiar vertical velocity gradients, 


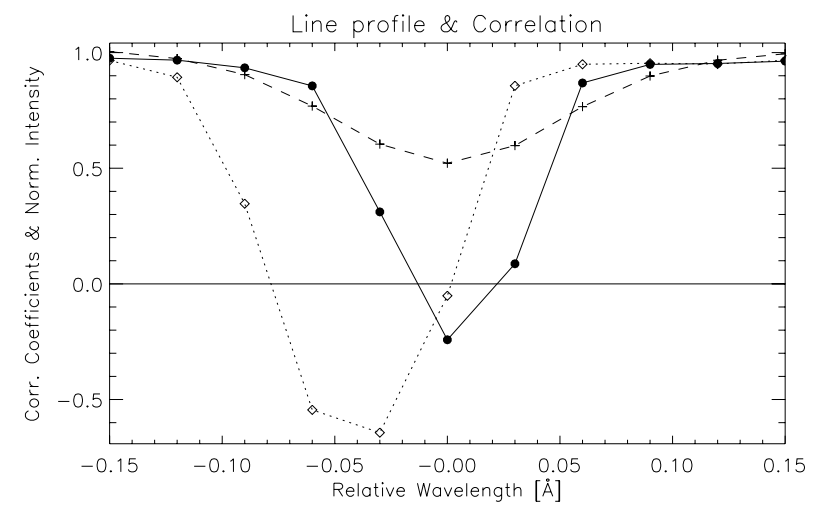

Fig. 7. Crosscorrelation coefficients between the continuum and the monochromatic images within the spectral line (dotted), showing a strong anticorrelation in the blue wing ("velocity cross-talk"). The solid line represents the same correlation coefficients after the spectral profiles were shifted to zero velocity. The average intensity profile of Fe I 7090.4 ̊̊ is given for reference (dashed line). Symbols indicate the spectral sampling.

or by insufficient spatial resolution (that might mix the signals of very different solar structures), symmetric spectral positions in the resampled lines should display the same (thermal) intensity patterns.

Figure 6 shows the resulting intensity maps for the same spectral positions as Fig. 5 (panels $a$ through $e$ ). Indeed, both pairs of images in the blue and red wing now exhibit essentially the same spatial patterns, with a correlation of 0.7 for the $\pm 60 \mathrm{~m} \AA$ images and 0.9 for $\pm 30 \mathrm{~m} \AA$. The images at $\pm 60 \mathrm{~m} \AA$ display the same low contrast, granular structures, while most of the intensity contrast fades in the inner wing images at $\pm 30 \mathrm{~m} \AA$. This should pinpoint the layer where temperature differences between granules and intergranules almost disappear. The line minimum intensity image (panel $c$ in Fig. 6) exhibits instead a fairly clear reversal of the granular intensity pattern. The solid line in Fig. 7 gives the cross-correlation values of these "at rest" images with the $7090 \AA$ continuum that are much more symmetric than before with respect to the line center. As seen also in the images, a significant anti-correlation is present only for the line minimum. While the plot refers to a single, representative scan, the average value of anticorrelation in the line center of all 171 scans was found to be $-0.21 \pm 0.05$. In spite of the low correlation coefficient, blinking the continuum and line center images clearly reveals the similarity of the intensity structures.

Panel $f$ in Fig. 6 provides a co-spatial, quasi-simultaneous image ( $\sim 10 \mathrm{~s}$ difference) acquired at Ca II $8542-0.9 \AA$. As the $\mathrm{Ca}$ II line has a very reduced velocity sensitivity at this wavelength, no further correction has been applied other than the standard reduction described in Sect. 2. Panel $f$ bears a striking resemblance to the Fe I line minimum intensities, derived in a completely independent fashion. Such an intensity pattern is also reminiscent of the high resolution $\mathrm{Ca}$ II $\mathrm{H} \& \mathrm{~K}$ wing images shown in Lites et al. (1999) and Rutten et al. (2004), providing strong confidence that our observations unambiguously present the signature of reversed granulation. The average correlation coefficient between the Ca II wing image and the $7090 \AA$ continuum over the whole dataset is $-0.32 \pm 0.07$, matching i.e. the value of -0.32 found by Rutten et al. (2004), who correlated $\mathrm{Ca}$ II $\mathrm{H}$ wing images with simultaneous $\mathrm{G}$ band images. The anticorrelation between $\mathrm{Ca}$ II and continuum is higher than between Fe I and continuum - the spatial pattern outlined in the Ca II wings mimics the reverse granular pattern more closely than the Fe I minimum intensity.

For the Ca II $8542 \AA$ line we derived even higher anticorrelation coefficients $(-0.43 \pm 0.06)$ when comparing images acquired at $\Delta \lambda=-0.6 \AA$ to $7090 \AA$ continuum intensities. However, images at this wavelength are visibly contaminated by chromospheric structures, such as strongly velocity-shifted filamentary features connecting magnetic patches, thus casting doubts on the significance of the correlation coefficient in this case.

\subsection{Spatial dependence}

The paper by Rutten et al. (2004) reports an increase of the anticorrelation between the Ca II H inner wing and G-band intensities once a $1.5^{\prime \prime}$ boxcar smoothing was applied to the data. We examined the effect of spatial smoothing to our images, and found that up to a smoothing of about $0.5^{\prime \prime}$ the crosscorrelation, averaged over the time series, did not change, while it decreased with further degradation: from -0.21 to -0.13 and -0.08 for the Fe I 7090 data, and from -0.32 to -0.29 and -0.24 for the Ca II 8542 data, when smoothed over $1.0^{\prime \prime}$ and $1.5^{\prime \prime}$. Spatial scales of up to $0.5^{\prime \prime}$ thus are an important contributor to the signal in our case.

Rutten et al. (2004), starting with data possessing a spatial resolution similar to ours, find an improvement of the anticorrelation when applying a spatial smoothing of $1.5^{\prime \prime}$, opposite to our result. We believe that this property stems from the rather large spectral passband utilized for their CaII $\mathrm{H}$ observations ( $1.35 \AA F W H M)$. Although they estimate the latter signature to be formed in the mid-photosphere, much as the Fe I 7090.4 A core, such a passband is bound to include intensity originating in higher layers, where the reverse granulation signal would be less relevant than other phenomena. Indeed, they argue that a consistent part of the intensity pattern that they observe is due to to the presence of gravity waves (see below). However, it is not clear why the correlation they obtain with the continuum is significantly higher than what we find using either the core of the Fe I line or the Ca II 8542 far wings, for a pattern that we identify as directly related to the underlying granulation pattern. Our data yields the same value of anti-correlation when using images obtained closer to the core of the Ca II $8542 \AA$ line $(-0.6 \AA)$ that are clearly "contaminated" by high velocity chromospheric structures. It will be worthwhile to investigate this issue further, for example by examining the effects of a reduced spectral resolution on the IBIS Ca II data.

\subsection{Temporal dependence}

As in the present study the correlation between the atmospheric layers sampled by the continuum and the narrowband images 


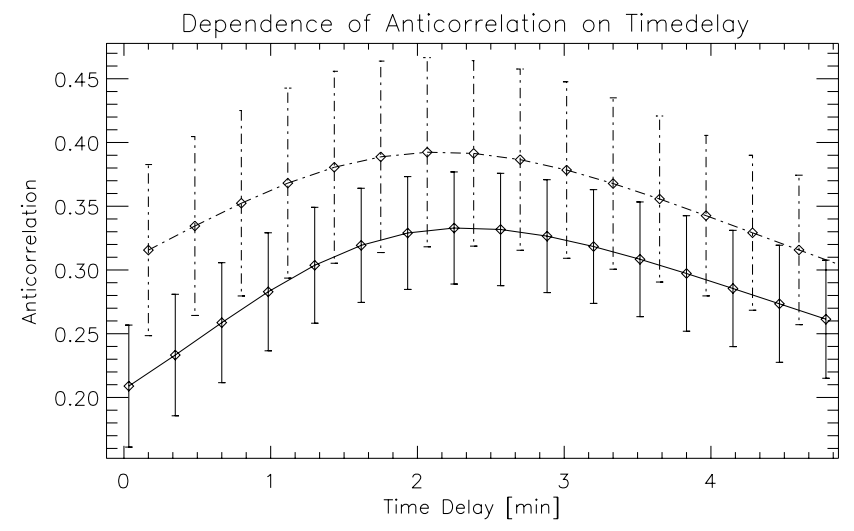

Fig. 8. Anti-correlation coefficients for the Fe I $7090 \AA$ Aine minimum and continuum intensities (solid lower line) and the Ca II $8542 \AA$ line wing and continuum intensities (dashed upper line), with varying time delay between continuum and spectral images. The values are obtained averaging over the whole time sequence; error bars give the standard deviation.

does not persist at larger scales, the correlation seems mostly related to the actual reversal of the temperature gradient between granules and intergranules, rather than to concurrent phenomena such as gravity waves. If this is the case, allowing a time delay between the correlated images should increase the agreement, as the convective overshoot depicted by the granular pattern at continuum heights will require a certain time to appear in the mid-photospheric layers sampled by the spectral lines. With a typical granular velocity of $1-2 \mathrm{~km} \mathrm{~s}^{-1}$ and an average height of $150-200 \mathrm{~km}$ for the layer where the temperature difference between granules and intergranules reverses, a delay of the order of 75-200s should provide the strongest negative crosscorrelation. This is indeed true for both, the Ca II wing and the Fe I minimum intensity, as shown in Fig. 8. The crosscorrelation coefficients, displayed with a negative sign, have been computed comparing continuum images with spectral images acquired at later times, and averaging results over the whole dataset. The strongest anticorrelation of $-0.39 \pm 0.07$ is found for a time delay of $124 \mathrm{~s}$ between the continuum and the Ca II wing image, which agrees with the findings of Rutten et al. (2004), and 135 s between the continuum and the Fe I line center image, giving an anticorrelation of $-0.33 \pm 0.04$.

The full temporal evolution of the anticorrelation coefficients between continuum and Fe I 7090 minimum intensities (upper panel) and Ca II 8542 wing (lower panel) is given in Fig. 9. The different curves refer to different time delays between continuum and line signatures, as described above, from 0 for the lower curves (stars) to $1 \mathrm{~min}$ (crosses) to $2 \mathrm{~min}$ (dots). The time delayed correlations were computed by keeping constant the mid-photospheric image and changing the continuum image, i.e. each spectral image was correlated with continuum images recorded at different, earlier times.

In addition to the increase of anticorrelation with delay already described, the Fe I curves exhibit a large temporal variation that looks clearly periodic. A power spectrum of these curves reveals a strong peak at about $6 \mathrm{~min}$. On the contrary, the Ca II correlation does not show any obvious periodicity - the power spectrum contains several peaks of equivalent

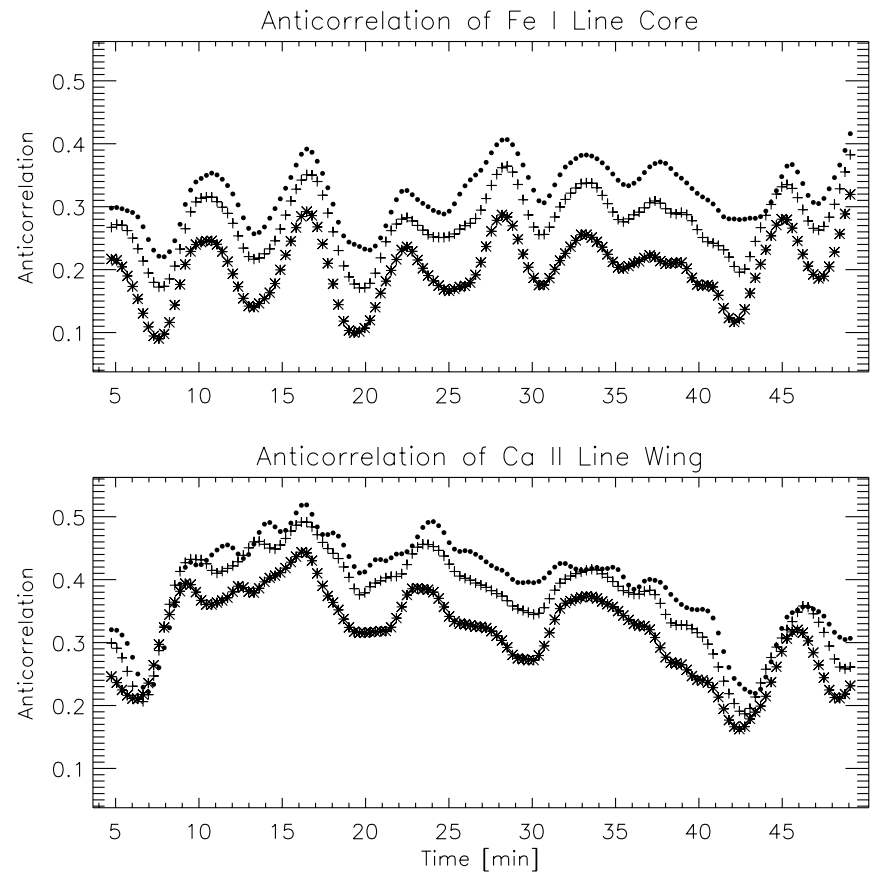

Fig. 9. Temporal evolution of anticorrelation values between Fe I $7090 \AA$ line core and continuum (top) and the Ca II $8542 \AA$ line wing and continuum (bottom). Different curves represent zero (stars), one minute (crosses) and two minute (dots) delay between continuum and spectral images.

amplitudes. Given its period, the oscillation in the Fe I correlation might look like a remnant of the $p$-mode filtering applied to the data, but it remains virtually unaltered after applying an even stronger subsonic filter with a cutoff at $c_{\mathrm{s}}=5 \mathrm{~km} \mathrm{~s}^{-1}$ that should remove most, if not all the power in the evanescent regime. The spatial smoothing to $1.0^{\prime \prime}$ and $1.5^{\prime \prime}$ described above also does not alter the amplitude of the oscillation. Since both the amplitude and the phase of the oscillation remain essentially constant for different delays, the oscillation has to be introduced in the upper level images, with little or no dependence on the changing continuum images. The same is true for the Ca II curve, although the variation is not periodic and more noisy in this case.

The higher values of anticorrelation found for the Ca II 8542 wing and the differences in temporal evolution (Figs. 8 and 9) can be framed in a coherent picture if we assume that the Ca II 8542 wing forms at lower levels than the Fe I 7090 core and that, at the height of formation of the Fe I 7090 core, a concomitant phenomenon, namely gravity waves, has developed to a clearly measurable level. Both assumptions are based on earlier works. On the one hand, the analysis of Uitenbroek (1989, 2004, private communication) shows that in a classical, semi-empirical model such as VAL-C, the wings of the Ca II line reach optical depth unity at a height of about $150 \mathrm{~km}$. As described in Sect. 2.3, the same approach yields optical depth unity for the core of the Fe I line around $200 \mathrm{~km}$. On the other hand, while gravity waves are easily excited by the convection overshoot in the stable photosphere, they are expected to develop steeply with height (Mihalas \& Toomre 1981) and should become readily visible only from the 
mid-photosphere upward (cf. introduction of Rutten \& Krijger 2003; Straus \& Bonaccini 1997). In this scenario we believe the height difference between Fe I line core and Ca II line wing to be enough to provide a detectable signature of gravity waves. They would "corrupt" the reverse granulation signal, causing the lower value of the anticorrelation with the continuum found for the core of Fe I $7090 \AA$, and explain its strong periodicity.

In order to test this hypothesis, in principle one could perform the same analysis on either Ca II images obtained closer to the line core (thus formed higher in the atmosphere) that should replicate the behavior of the Fe I 7090 core, or Fe I images obtained in the inner wing that should replicate the behaviour of the Ca II 8542 wing. However within the constraints of our simplified analysis, neither of these options are viable. As stated before, the Ca II line acts as a two-steps function, with extended wings sampling the low-mid photosphere and a narrow range of wavelengths around the core formed in the mid-chromosphere, with little diagnostic power at intermediate heights. In particular, images acquired at about $0.6 \AA$ from the line core show a clear superposition of (magnetic) chromospheric structures on top of photospheric ones, rendering meaningless a direct attempt to correlate them with the continuum. For the Fe I 7090 line, while the core forms at or above $200 \mathrm{~km}$, and the outer wings form essentially at continuum level, the inner wings are formed over a large range in the photosphere with an almost flat distribution (Asensio Ramos et al. 2006). Therefore, images acquired at these wavelengths mix information originating in layers with quite different physical properties, making a direct comparison with the Ca II wing images difficult. Such limitations could be overcome by a full inversion of the radiative transfer equation for both lines (e.g. Socas-Navarro et al. 1998, 2000) that could retrieve a fair estimation of the atmospheric parameters even at low-sensitivity heights, but this exceeds the scope of this paper.

\section{Conclusions}

We have presented a first investigation of the quiet photosphere performed with the tunable, double Fabry-Perot Interferometric BI-dimensional Spectrometer (IBIS, Cavallini 2006), recently installed at the NSO/DST. The narrow spectral passband of IBIS allows for a fine spectral sampling with high temporal and spatial resolution. We analyzed a $1 \mathrm{hr}$ series of a very quiet internetwork region from good quality observations in the mid-photospheric Fe I 7090.4 $\AA$ line, the deep-photospheric Fe II $7224.5 \AA$ line and the wing of Ca II $8542 \AA$.

Velocities. The intensity and velocity distributions for Fe I and Fe II are consistent with the literature values. The correlation between the center of gravity and line core velocities of both lines is very high, giving a better value for smaller height differences. Hence, the velocity pattern maintains its identity throughout the mid-photosphere. As expected, the velocity pattern displays a large anticorrelation with the continuum intensities. An especially good correlation, as well as comparable amplitudes, are found between the COG and line core velocities of the $7090 \AA$ line, confirming the equivalence of the core and COG velocity values for low atmospheric lines (Uitenbroek 2003). A first inspection of granular versus intergranular behaviour with height is consistent with the picture of steeply decelerating intergranular velocities predicted by twocomponent models of the lower solar atmosphere (Borrero \& Bellot Rubio 2002).

Reversed granulation. The photospheric Fe I and the Ca II line wing are used to study the "reversed granulation", the partial reversal of the continuum intensity pattern in the low- or mid-photosphere, predicted by numerical simulations, and often visualized in the extended wings of Ca II H \& K (Evans \& Catalano 1972; Lites et al. 1999; Rutten et al. 2004).

The Fe I line clearly shows the transition between granular intensity structure in the wings to a reversed intensity structure in the line core. To make this phenomenon readily apparent, we must take into account the vertical velocity at each position, including $p$-mode effects. The narrow passband of the interferometer plays an equally important role, as the pattern is visible only in line center and disappears only $30 \mathrm{~m} \AA$ further in the wings. Quasi-simultaneous observations in the blue wing of the Ca II line, unaffected by velocity, reveal a very similar reversed granulation pattern, but give a higher anti-correlation value with the continuum. The low correlation coefficients are consistent with expectations of numerical simulations in the mid-photosphere (Nordlund 1985).

For both signatures the anti-correlation with the continuum remains constant with spatial smoothing up to about $0.5^{\prime \prime}$, but in contradiction to Rutten et al. (2004), rapidly decreases if a lower resolution is allowed. This indicates that the observed phenomenon of reversed granulation has the typical granulation scale, including narrow intergranular lanes. In agreement with Rutten et al. (2004), we find an increase in the anticorrelation if a time delay is allowed between the compared images. The strongest anti-correlation between mid-photospheric intensities and continuum occurs for a time delay of $\sim 2 \mathrm{~min}$. These properties are consistent with the idea of the reversed granulation pattern beeing mostly determined by adiabatic expansion cooling of the overshooting plasma.

Oscillations. The temporal evolution of the anticorrelation between continuum and Fe I line center intensities shows an oscillatory signal with a period of about $6 \mathrm{~min}$, originating in the mid-photosphere. In the temporal evolution of the Ca II wing correlation with the continuum, no clear periodic signal can be detected. Spatial smoothing alters the values of the correlation coefficients, but not the signature of the oscillation, implying that the phenomenon is coherent over greater than granular scales. Finally, the signal persists even after the application of a stronger subsonic filter that should guarantee a complete removal of the oscillatory power in the evanescent regime.

These findings are consistent, given that the CaII wing images form below the Fe I line core (Uitenbroek 1989) and that gravity waves develop steeply with height in the midphotosphere (Mihalas \& Toomre 1981). The period of the oscillation and its presence only in the higher formed iron line core, together with the anti-correlation with the continuum stronger at the lower layers sampled by the Ca II wing, is therefore suggestive of the presence of gravity waves in the mid photosphere. The reversed granulation pattern, quite strong in the Ca II images and peaking at the spatial scale of granulation, 
becomes corrupted in the Fe I core by the effect of gravity wave oscillations that start in the mid-photosphere. We plan to perform a more detailed study of this issue in a forthcoming work, including proper $k-\omega$ - power and phase difference diagrams.

Acknowledgements. We thank T. Rimmele, K. Reardon and M. Woodward for the use of their observations at the DST/NSO and the observing assistents M. Bradford, D. Gilliam and J. Elrod for their support. The National Solar Observatory is operated by the Association of Universities for Research in Astronomy, Inc. (AURA), under cooperative agreement with the National Science Foundation. The IBIS instrument was designed and developed by F. Cavallini, INAF Arcetri. We thank K. Reardon for help with the data. We acknowledge A. Falchi for fruitful discussions, M. Carlsson for calculation of response functions and A. de Wijn for his scatterplot program. We thank the referee J. M. Borrero for his detailed comments. This research was funded through the European Solar Magnetism Network (ESMN, contract HPRN-CT-2002-00313).

\section{References}

Al, N., Bendlin, C., \& Kneer, F. 2002, A\&A, 383, 283

Asensio Ramos, A., Janssen, K., Cauzzi, G., \& Reardon, K. 2006, A\&A, in preparation

Asplund, M., Nordlund, A., Trampedach, R., Allende Prieto, C., \& Stein, R. 2000a, A\&A, 359, 729

Asplund, M., Nordlund, A., Trampedach, R., \& Stein, R. F. 2000b, A\&A, 359, 743

Berrilli, F., Consolini, G., Pietropaolo, E., et al. 2002, A\&A, 381, 253

Borrero, J. M., \& Bellot Rubio, L. R. 2002, A\&A, 385, 1056

Cabrera Solana, D., Bellot Rubio, L. R., \& del Toro Iniesta, J. C. 2005 , A\&A, 439, 687

Cauzzi, G., Smaldone, L. A., Balasubramaniam, K. S., \& Keil, S. L. 1993, Sol. Phys., 146, 207

Cauzzi, G., Janssen, K., Cavallini, F., \& Reardon, K. 2006, Kluwer, Jenam 2004 Proceedings, in press

Cavallini, F. 2002, in ESA SP-477: Solspa 2001, Proc. of the Second Solar Cycle and Space Weather Euroconference, ed. H. Sawaya-Lacoste, 585

Cavallini, F. 2006, Sol. Phys., in press

Domínguez Cerdeña, I., Kneer, F., \& Sánchez Almeida, J. 2003, ApJ, 582, L55
Espagnet, O., Muller, R., Roudier, T., Mein, N., \& Mein, P. 1995, A\&AS, 109, 79

Evans, J. W., \& Catalano, C. P. 1972, Sol. Phys., 27, 299

Hanslmeier, A., Kučera, A., Rybák, J., Neunteufel, B., \& Wöhl, H. 2000, A\&A, 356, 308

Hirzberger, J. 2002, A\&A, 392, 1105

Janssen, K. 2003, Ph.D. Thesis, Univ.-Sternwarte Göttingen

Keil, S. L., \& Canfield, R. C. 1978, A\&A, 70, 169

Krieg, J., Kneer, F., Koschinsky, M., \& Ritter, C. 2000, A\&A, 360, 1157

Lites, B. W., Rutten, R. J., \& Berger, T. E. 1999, ApJ, 517, 1013

Löfdahl, M. G. 2002, in Image Reconstruction from Incomplete Data II, ed. P. J. Bones, M. A. Fiddy, \& R. P. Millane, Proc. SPIE, 4792, 146

Mihalas, B. W., \& Toomre, J. 1981, ApJ, 249, 349

Nesis, A., Hammer, R., Kiefer, M., et al. 1999, A\&A, 345, 265

Nordlund, A. 1985, Sol. Phys., 100, 209

Peter, H., Gudiksen, B. V., \& Nordlund, Å. 2004, ApJ, 617, L85

Puschmann, K., Vázquez, M., Bonet, J. A., Ruiz Cobo, B., \& Hanslmeier, A. 2003, A\&A, 408, 363

Rimmele, T. R. 2004, in Advancements in adaptive optics., ed. D. B. Calia, B. L. Ellerbroek, \& R. Ragazzoni, Proc. SPIE, 5490, 34

Rodríguez Hidalgo, I., Ruiz Cobo, B., Collados, M., \& del Toro Iniesta, J. C. 1999, in Stellar Structure: Theory and Test of Connective Energy Transport, ASP Conf. Ser., 173, 313

Rutten, R. J., \& Krijger, J. M. 2003, A\&A, 407, 735

Rutten, R. J., de Wijn, A. G., \& Sütterlin, P. 2004, A\&A, 416, 333

Salucci, G., Bertello, L., Cavallini, F., Ceppatelli, G., \& Righini, A. 1994, A\&A, 285, 322

Shchukina, N., \& Trujillo Bueno, J. 2001, ApJ, 550, 970

Socas-Navarro, H., Ruiz Cobo, B., \& Trujillo Bueno, J. 1998, ApJ, 507,470

Socas-Navarro, H., Trujillo Bueno, J., \& Ruiz Cobo, B. 2000, ApJ, 530, 977

Stein, R. F., \& Nordlund, A. 1998, ApJ, 499, 914

Straus, T., \& Bonaccini, D. 1997, A\&A, 324, 704

Sütterlin, P., Rutten, R. J., \& Skomorovsky, V. I. 2001, A\&A, 378, 251

Uitenbroek, H. 1989, A\&A, 213, 360

Uitenbroek, H. 2003, ApJ, 592, 1225

Wedemeyer, S., Freytag, B., Steffen, M., Ludwig, H.-G., \& Holweger, H. 2004, A\&A, 414, 1121 